\title{
Single-Incision Laparoscopic Splenectomy for an Unruptured Aneurysm of the Splenic Artery
}

\author{
Yoshitaka Toyoda Tsuyoshi Igami Yosuke Ochiai Tomoki Ebata \\ Yukihiro Yokoyama Gen Sugawara Takashi Mizuno Junpei Yamaguchi \\ Masato Nagino
}

Division of Surgical Oncology, Department of Surgery, Nagoya University Graduate School of Medicine, Nagoya, Japan

\section{Significance of the Study}

- Single-incision laparoscopic surgeries for splenic disorders are recognized as challenging procedures and remain controversial. Single-incision laparoscopic splenectomy is a suitable and safe procedure for treatment of an unruptured aneurysm of the splenic artery that is close to the hilum of the spleen without splenomegaly when transcatheter arterial embolization cannot be performed.

\section{Keywords}

Single-incision laparoscopic splenectomy · Aneurysm of the splenic artery · Laparoscopic splenectomy

\footnotetext{
Abstract

Objective: We describe our experience of single-incision laparoscopic splenectomy (SILS) for an unruptured aneurysm of the splenic artery. Clinical Presentation and Intervention: $A$ 73-year-old woman was diagnosed as having a splenic aneurysm which grew from 14 to $22 \mathrm{~mm}$ in diameter within 2 years. Due to a contrast agent allergy, transcatheter arterial embolization could not be performed; therefore, SILS was performed with a 4-cm Z-shaped incision. The operative time and intraoperative blood loss were $132 \mathrm{~min}$ and $27 \mathrm{~mL}$, respectively. The patient was discharged 4 days after surgery. Conclusion: In selected cases, SILS is a suitable and safe procedure for an unruptured aneurysm of the splenic artery.

(c) 2017 The Author(s)

Published by S. Karger AG, Basel
}

\section{KARGER}

E-Mail karger@karger.com www.karger.com/mpp

\section{The Author(s) \\ Published by S. Karger AG, Basel \\ Karger \\ Open access}

This is an Open Access article licensed under the Creative Commons Attribution-NonCommercial-4.0 International License (CC BY-NC) (http://www.karger.com/Services/OpenAccessLicense), applicable to the online version of the article only. Usage and distribution for commercial purposes requires written permission.

\section{Introduction}

Transcatheter arterial embolization is recognized as a suitable procedure for unruptured visceral artery aneurysm $[1,2]$. When transcatheter arterial embolization is impossible, a surgical approach is required to treat an unruptured visceral artery aneurysm [3]. Laparoscopic surgery for unruptured visceral artery aneurysm has been increasingly reported $[4,5]$. However, single-incision laparoscopic surgeries for splenic disorders are recognized as challenging procedures and remain controversial. Hence, we describe our experience with single-incision laparoscopic splenectomy (SILS) in a patient with an unruptured aneurysm of the splenic artery on whom a transcatheter arterial embolization could not be performed. 


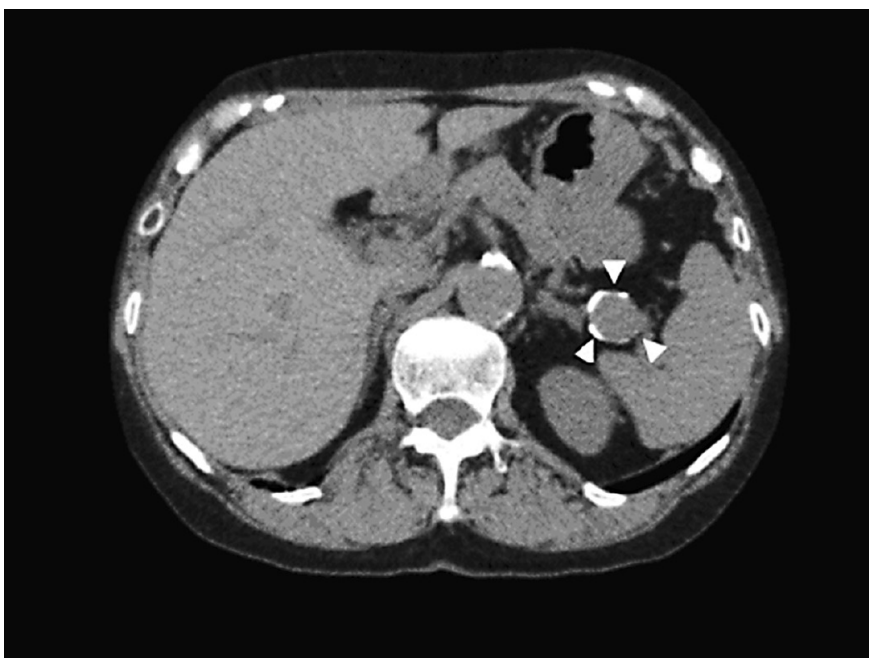

Fig. 1. Preoperative computed tomography revealed that the aneurysm of the splenic artery (arrowheads), measuring $22 \mathrm{~mm}$ in diameter, separated from the tail of the pancreas, was close to the hilum of the spleen without splenomegaly.

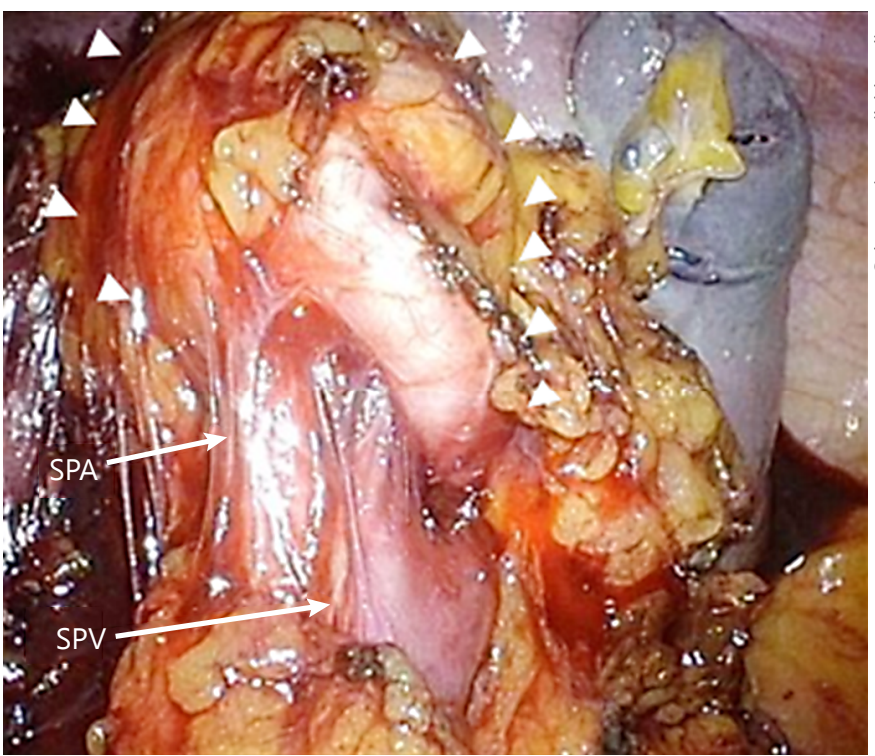

Fig. 3. Intraoperative findings of the aneurysm of the splenic artery. Laparoscopic findings revealed an unruptured aneurysm of the splenic artery (arrowheads). It was difficult to divide the splenic artery from the splenic vein. SPA, splenic artery; SPV, splenic vein.

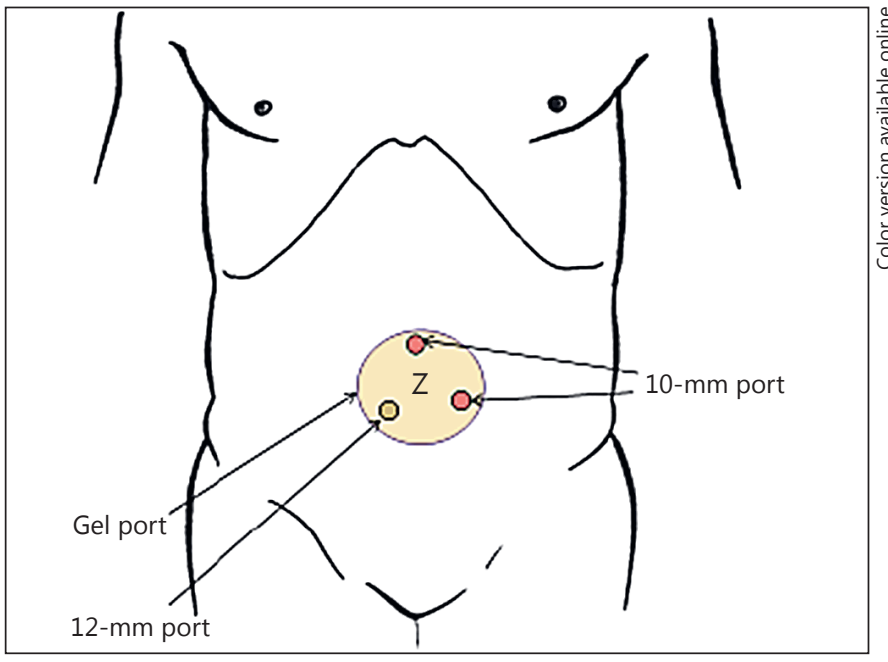

Fig. 2. Schema of the port position. A Z-shaped skin incision (4 $\mathrm{cm}$ ) was made in the umbilical region and a gel port (GelPOINT Advanced Access Platforms ${ }^{\circledR}$; Applied Medical Resources Corp.) was placed through the umbilical incision with a $12-\mathrm{mm} \mathrm{Hg}$ pneumoperitoneum using carbon dioxide. A 12-mm and two $10-\mathrm{mm}$ ports were placed through the gel port. A 12-mm flexible scope was inserted through the $12-\mathrm{mm}$ port to explore the abdominal cavity.

\section{Case Report}

A 73-year-old woman was diagnosed 2 years ago as having an unruptured aneurysm of the splenic artery, measuring $14 \mathrm{~mm}$ in diameter. She had not undergone abdominal surgery. Recently, the aneurysm became enlarged to $22 \mathrm{~mm}$ in diameter (Fig. 1). She had no symptoms and her BMI was 21.0. Due to a contrast agent allergy, transcatheter arterial embolization could not be performed; therefore, a surgical approach was required. Computed tomography revealed that the aneurysm was separated from the tail of the pancreas and close to the hilum of the spleen without splenomegaly (Fig. 1). Consequently, SILS was planned.

The surgical procedures were as follows: the patient was placed in the reverse Trendelenburg position $[4,5]$. Then, a Z-shaped skin incision was made in the umbilical region and a gel port (GelPOINT Advanced Access Platforms ${ }^{\circledR}$; Applied Medical Resources Corp., Rancho Santa Margarita, CA, USA) was placed through the umbilical incision with a $12-\mathrm{mm} \mathrm{Hg}$ pneumoperitoneum using carbon dioxide (Fig. 2) [6]. A 12-mm and two 10-mm ports were placed through the gel port (Fig. 2). A 12-mm flexible scope (ENDOEYE FLEX ${ }^{\circledR}$ LTF-S190-10; Olympus Corp., Tokyo, Japan) was inserted through the $12-\mathrm{mm}$ port to explore the abdominal cavity. After separating the greater omentum from the spleen by using articulated laparoscopic coagulating shears (ENSEAL ${ }^{\circledR}$ G2 Articulating Tissue Sealers; Ethicon, USA) and articulated laparoscopic forceps (SILS ${ }^{\mathrm{TM}}$ Articulating Hand Instruments, DISSECTOR XL; Covidien, Medtronic, Inc., USA). Laparoscopic findings revealed an unruptured aneurysm of the splenic artery was separated from the pancreas (Fig. 3). Encircling the splenic artery and vein together was easy, but dividing the splenic artery from the splenic vein 


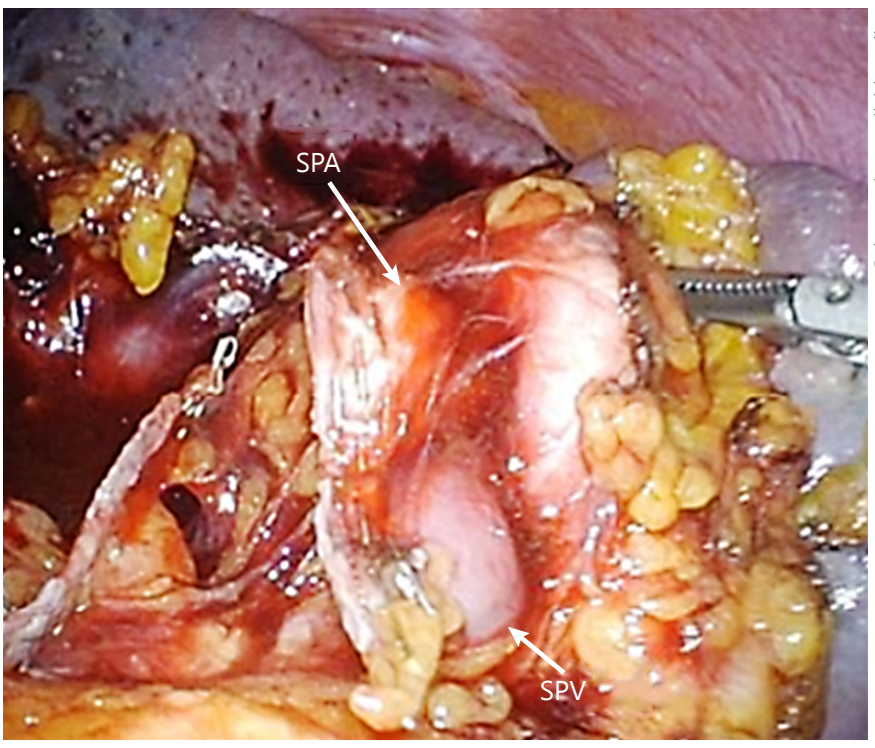

Fig. 4. Intraoperative findings after cutting the splenic artery and vein. The splenic artery and vein were cut together using an articulated endoscopic linear stapler (Endo GIA ${ }^{\mathrm{TM}}$, Tri-Staple ${ }^{\mathrm{TM}}$; Covidien, Medtronic, Inc.). SPA, splenic artery; SPV, splenic vein.

was difficult; therefore, using an articulated endoscopic linear stapler (Endo GIA ${ }^{\mathrm{TM}}$, Tri-Staple ${ }^{\mathrm{TM}}$; Covidien, Medtronic, Inc.), the splenic artery and vein were cut together (Fig. 4). After placing the resected specimen inside the retrieval bag, the spleen was crushed in the bag and the resected specimen was removed through the Zshaped umbilical incision. After irrigation of the abdominal cavity, the umbilical incision was carefully closed without the use of any drainage tubes. The operative time and intraoperative blood loss were $132 \mathrm{~min}$ and $27 \mathrm{~mL}$, respectively.

The macroscopic findings of the resected specimen showed an aneurysm while histological examination revealed a true aneurysm. The total length of the Z-shaped incision was $4 \mathrm{~cm}$. The patient was discharged from our hospital on day 4 with no postoperative complications and was quite satisfied with the cosmetic result 8 months after the surgery.

All human studies have been reviewed by the appropriate ethics committee and were performed in accordance with the ethical standards laid down in an appropriate version of the Declaration of Helsinki. Informed consent was obtained from the subject prior to inclusion in this study.

\section{Discussion}

In this case, transcatheter embolization could not be performed for treatment of an unruptured aneurysm of the splenic artery because of an allergy to contrast agent. A surgical approach was required. As a minimally invasive treatment, SILS was successful, with no complications.
Although transcatheter arterial embolization is widely accepted as a suitable and less invasive procedure for unruptured visceral artery aneurysms $[1,2]$, it is occasionally impossible for certain reasons, e.g., contrast agent allergy or technical problems. In such situations, a surgical approach is a necessary and reliable procedure [3-5]. Laparoscopic surgery has become increasingly accepted as a surgical approach for unruptured visceral artery aneurysms in which transcatheter arterial embolization is impossible $[4,5]$. When the aneurysm of the splenic artery is close to the hilum of the spleen, laparoscopic splenectomy is a suitable and safe procedure [3-5, 7]. In previous reports $[3-5,7,8]$, laparoscopic splenectomies for aneurysms of the splenic artery were performed in 24 patients, including 3 patients with a distal pancreatectomy. SILS for aneurysms of the splenic artery were performed in 3 patients, including our patient; however, 2 other patients had an additional port and/or a cloth tape to hang the hilum of the spleen from an extra-abdominal cavity [8]. To our knowledge, this is the first successful case of a "pure" SILS for treatment of an unruptured aneurysm of the splenic artery. We utilized a Z-shaped umbilical incision and a gel port to obtain a wide entrance into the abdominal cavity [6]; therefore, we obtained easy and excellent manipulability despite SILS. Consequently, SILS for an unruptured aneurysm of the splenic artery was achieved with a successful outcome.

According to previous reports regarding SILS $[9,10]$, venous bleeding is an important factor in the decision to convert to open surgery. To prevent bleeding from the short gastric vein during laparoscopic splenectomy, the use of an energy device is recommended $[3-5,9,10]$. Because the manipulability of a straight energy device is inconvenient during SILS, we used articulated laparosonic coagulating shears and articulated laparoscopic forceps. We could thus safely separate the greater omentum from the spleen. To prevent bleeding from the splenic vein and/or the hilum of the spleen during laparoscopic splenectomy, it is recommended that the splenic artery and vein be cut together using an endoscopic linear stapler without labored separation $[3-5,9,10]$. We could cut the splenic artery and vein together using an articulated laparoscopic linear stapler. Achievement of SILS for an unruptured aneurysm of the splenic artery requires the use of appropriate instruments. Additionally, splenomegaly is an important factor in the decision to convert to open surgery $[9,10]$; therefore, SILS for aneurysm of the splenic artery should be used in selected patients without splenomegaly. 


\section{Conclusion}

In selected patients, SILS is a suitable and safe procedure for excision of the unruptured aneurysm of the splenic artery that is close to the hilum of the spleen without splenomegaly when transcatheter arterial embolization cannot be performed.

\section{Disclosure Statement}

The authors have no conflict of interests or financial ties to disclose.

\section{References}

1 Belli AM, Markose G, Morgan R: The role of interventional radiology in the management of abdominal visceral artery aneurysms. Cardiovasc Intervent Radiol 2012;35:234-243.

2 Tulsyan N, Kashyap, Greenberg RK, et al: The endovascular management of visceral artery aneurysms and pseudoaneurysms. J Vasc Surg 2007;45:276-283.

$>3$ Wang CX, Han LN, Liang FQ, et al: Aneurysm resection and vascular reconstruction for true aneurysm at the initial segment of splenic artery. J Huazhong Univ Sci Technolog Med Sci 2015;35:439-444.
4 Pietrabissa A, Ferrari M, Berchiolli R, et al: Laparoscopic treatment of splenic artery aneurysms. J Vasc Surg 2009;50:275-279.

5 Iida A, Katayama K, Yamaguchi A: Laparoscopic resection for splenic artery aneurysm using the lateral approach: report of two cases. Asian J Endosc Surg 2013;6:147-150.

6 Igami T, Tsuchiya T, Ebata T, et al: Application of a Z-shaped umbilical incision and a saline-cooled radiofrequency device to singleincision laparoscopic surgery for a huge liver cyst: report of a case. Int Surg 2015;100:10981103.
7 Toyoda Y, Kyokane T, Kawai T, et al: Laparoscopic surgery for the treatment of a ruptured aneurysm of the right gastric artery: a case report. Asian J Endosc Surg 2017;10:70-74.

8 Misawa T, Sakamoto T, Ito R, et al: Singleincision laparoscopic splenectomy using the "tug-exposure technique" in adults: results of ten initial cases. Surg Endosc 2011;25:32223227.

$\checkmark 9$ Gkegkes ID, Mourtarakos S, Iavazzo C: Single-incision laparoscopic splenectomy. JSLS 2014;18:e2014.00350.

10 Fan Y, Wu SD, Kong J, et al: Feasibility and safety of single-incision laparoscopic splenectomy: a systematic review. J Surg Res 2014; 186:354-362. 